\title{
Application of low anterior mediastinal tracheostomy for locally advanced cervicothoracic esophageal cancer undergoing total laryngopharyngoesophagectomy: a case report
}

\author{
Chunji Chen $^{1}$, Raja Flores ${ }^{2}$, Biniam Kidane ${ }^{3}$, Masatsugu Hamaji ${ }^{4}$, Xufeng Guo ${ }^{1}$ \\ ${ }^{1}$ Department of Thoracic Surgery, Shanghai Chest Hospital, Shanghai Jiaotong University, Shanghai, China; ${ }^{2}$ Department of Thoracic Surgery, \\ Icahn School of Medicine at Mount Sinai, Mount Sinai Health System, New York, NY, USA; ${ }^{3}$ Section of Thoracic Surgery, University of Manitoba, \\ Winnipeg, Manitoba, Canada; ${ }^{4}$ Department of Thoracic Surgery, Kyoto University Hospital, Kyoto, Japan \\ Correspondence to: Xufeng Guo. Department of Thoracic Surgery, Shanghai Chest Hospital, Shanghai Jiaotong University, 241 West Huaihai Road, \\ Shanghai 200030, China. Email: shandagxf@126.com.
}

\begin{abstract}
A sixty-six year-old woman came to hospital, complaining of dysphagia and weight loss. Esophagoscope showed a neoplasm between 15 and $20 \mathrm{~cm}$ from the incisors, biopsy revealed esophageal squamous cell carcinoma. Chest computed tomography (CT) showed that the cervical esophageal wall became thicker, the narrowing of the lumen extended downwards to the upper thoracic esophagus. Tumor invaded the membranous parts of the 5 th to 12 th rings of the trachea, and no swollen lymph nodes were observed in the mediastinum. The clinical stage was cT4N0M0 with borderline resectable possibility. The patient was assessed with stable disease after receiving two courses of neoadjuvant chemotherapy using DTX/CDDP/5-FU strategy. After a standard multidisciplinary treatment evaluation, total laryngopharyngoesophagectomy plus low anterior mediastinal tracheostomy (AMT) was performed. To ensure radical resection, we innovatively adopted the application of Double S-shaped myocutaneous flaps, which helps to extend the trachea and facilitate the stoma. Meanwhile, we removed the upper sternum, sternum stem, clavicle head and first and second costal cartilage to facilitate the tracheostomy and reduce the space around the stoma to avoid fluid accumulation. The pathological result showed $\mathrm{pT}_{4 \mathrm{~b}} \mathrm{~N}_{0} \mathrm{M}_{0}$ stage with partial response after neoadjuvant chemotherapy. This patient developed a minor anastomotic leakage which was effectively managed. Finally, she was discharged successfully 21 days after surgery.
\end{abstract}

Keywords: Esophageal neoplasma; neoadjuvant therapy; esophagectomy; case report

Submitted Jul 14, 2021. Accepted for publication Dec 15, 2021.

doi: 10.21037/jgo-21-685

View this article at: https://dx.doi.org/10.21037/jgo-21-685

\section{Introduction}

At the cervicothoracic junction, the esophagus has a close relationship with the surrounding organs including trachea, great vessels and recurrent laryngeal nerve due to the narrow space. Locally advanced esophageal cancer in this area may often easily invade these organs, especially the trachea. Therefore, radical resection of locally advanced esophageal cancer in the cervicothoracic junction is technique demanding. The standard treatment for primary clinical stage $T_{4 b}$ tumor with tracheal invasion is definitive chemoradiotherapy (dCRT). However, there are still some patients with residual tumor or recurrence even after receiving dCRT. Salvage surgery after dCRT has been reported to improve the survival of such patients, and is considered an alternative treatment option (1-4). The reasons for choosing induction therapy rather than dCRT are as follows:

(I) The initial clinical stage was $c \mathrm{~T}_{4} \mathrm{~N}_{0} \mathrm{M}_{0}$ with borderline resectable possibility and negative $\mathrm{cN}$ stage. There is possibility of surgery for this patient.

(II) There is a higher postoperative complication rate for the patients who have received dCRT, especially 

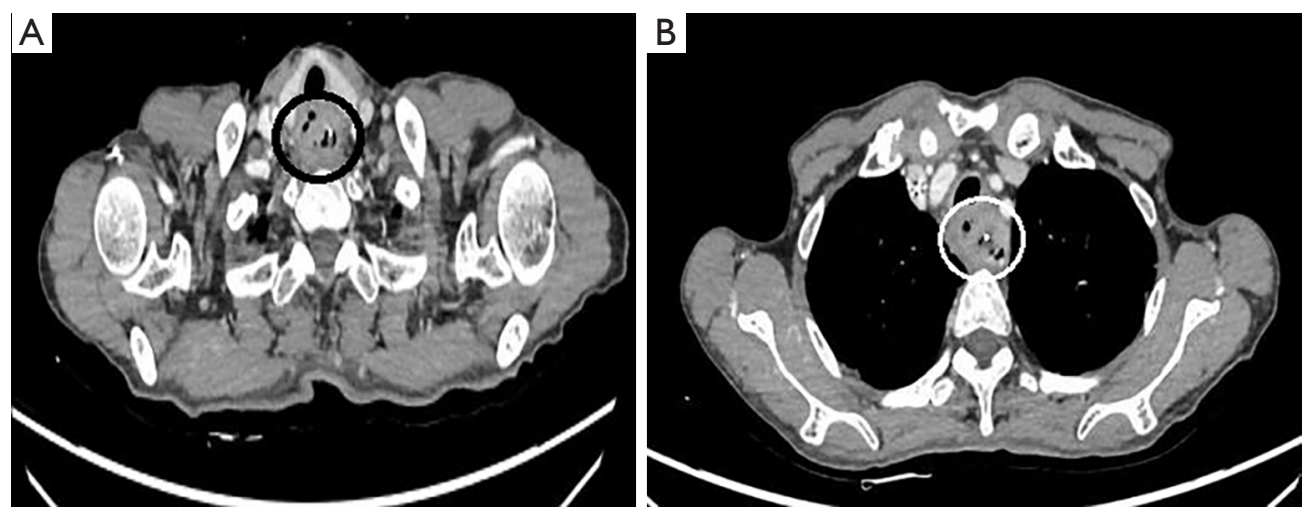

Figure 1 After induction chemotherapy, CT revealed multiple air spaces were visible at the site of the primary lesion (circled sign), and there was no obvious tumor regression.

respiratory complication.

(III) Although dCRT might bring better remission of tumor than chemotherapy only, we chose DCF (docetaxel, cisplatin, and 5-fluorouracil) threedrug chemotherapy regimen because we could use radiotherapy in the future if the efficacy of DCF was not good.

However, salvage surgery is associated with a high risk of postoperative complications, especially for patients who accepted total laryngopharyngoesophagectomy combined with low anterior mediastinal tracheostomy (AMT). Here we share a case of conversion surgery for cervicothoracic esophageal cancer involving a long trachea. In order to obtain a radical resection, low AMT was conducted. We present the following article in accordance with the CARE reporting checklist (available at https://dx.doi.org/10.21037/ jgo-21-685).

\section{Case presentation}

The 66-year-old woman came to our hospital, complaining of dysphagia for over one month. Esophagoscope found a squamous cell carcinoma between 15 and $20 \mathrm{~cm}$ from the incisors. The esophagus was involved for all its circumferences. An irregular ridge-like, centrally ulcerated lesion was visible. The lumen was narrow, barely allowing the passing of a thin endoscope. No abnormality was found in other parts of the esophagus and the stomach. CT suggested a space-occupying lesion at the cervical and upper thoracic esophagus, with apparent narrowing of the nearby lumen. No enlarged lymph nodes were observed in the mediastinum, and the tumor was closely related to the tracheal membrane. Tracheoscopy showed that the lumen was visible, and the mucosa was smooth. PET-CT showed no distant metastasis. She had no symptoms including dyspnea and hoarseness. Specialist physical examinations showed no abnormality, and her cardiopulmonary function was normal. The patient had a previous history of tongue cancer resection. All procedures performed in this study involving human participants were in accordance with the ethical standards of the institutional and/or national research committee(s) and with the Helsinki Declaration (as revised in 2013). Written informed consent was obtained from the patient for publication of this case report and accompanying images. A copy of the written consent is available for review by the editorial office of this journal.

\section{Treatments}

The patient received two courses of three-drug neoadjuvant chemotherapy with docetaxel $\left(70 \mathrm{mg} / \mathrm{m}^{2}, 1 \mathrm{~h}\right.$ intravenous infusion on day 1$)$, cisplatin $\left(70 \mathrm{mg} / \mathrm{m}^{2}, 2 \mathrm{~h}\right.$ intravenous infusion on day 1), and 5 -fluorouracil $\left(750 \mathrm{mg} / \mathrm{m}^{2}, 24 \mathrm{~h}\right.$ continuous intravenous infusion on days $1-5)$ repeated every 3 weeks. No serious adverse events occurred during the neoadjuvant therapy.

One month later, the patient was readmitted for preoperative evaluation. After neoadjuvant chemotherapy, CT revealed the location of the lesion showed a chemotherapy-induced change. Multiple air spaces were visible at the primary lesion site, and there was no obvious tumor regression (Figure 1). Esophagoscopy suggested chemotherapy-induced changes at 15 to $20 \mathrm{~cm}$ from the incisors, and a neoplasm with ulcers was still visible. A rupture was suspected at $20 \mathrm{~cm}$ from the incisors, with occasionally emerging bubbles, suggesting a possible 


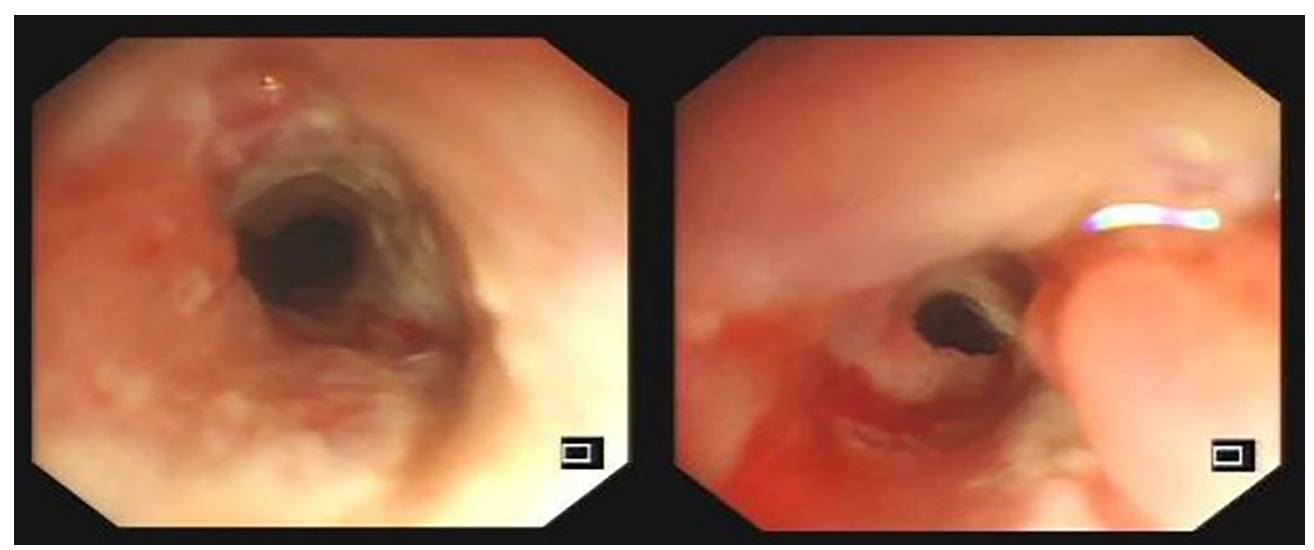

Figure 2 After induction chemotherapy, esophagoscopy suggested chemotherapy-induced changes at 15 to $20 \mathrm{~cm}$ from the incisors, and a neoplasm with ulcers was still visible. A rupture was suspected at $20 \mathrm{~cm}$ from the incisors, with occasionally emerging bubbles, suggesting a possible esophageal fistula.

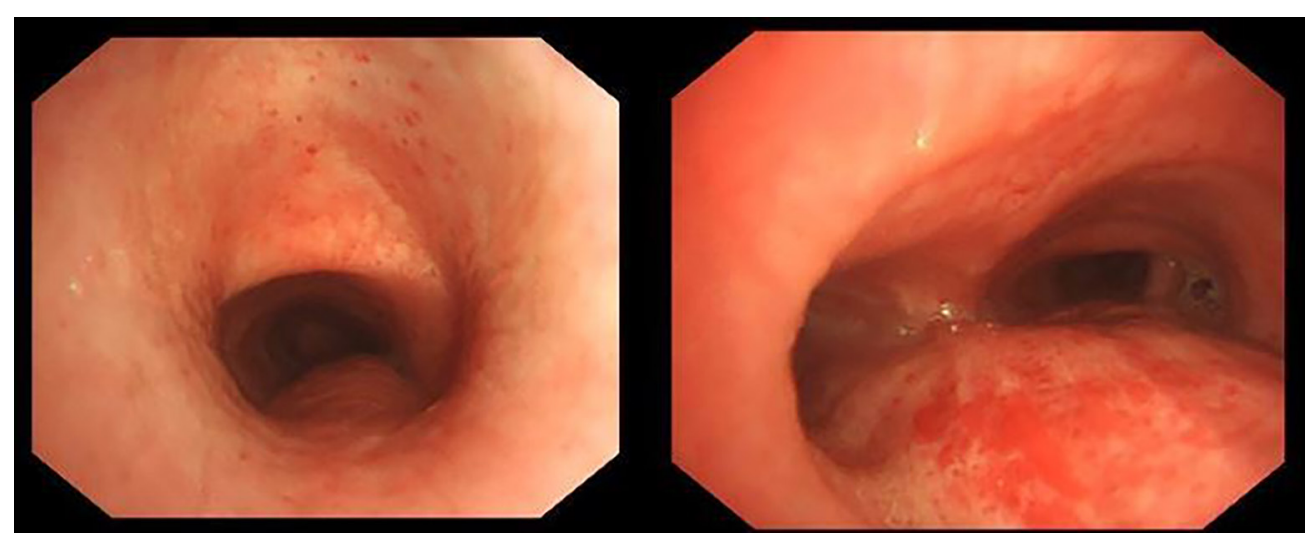

Figure 3 Tracheoscopy revealed that the mucosa of the tracheal membrane about $5 \mathrm{~cm}$ above the tracheal carina was significantly swollen, and the nearby lumen was narrow and became uneven starting from the infraglottic cavity.

esophageal fistula (Figure 2). Tracheoscopy revealed that the mucosa of the tracheal membrane about $5 \mathrm{~cm}$ above the tracheal carina was significantly swollen, and the nearby lumen was narrow (Figure 3).

After the informed consent was obtained from the patient and her family, an individualized therapeutic strategy was designed. Total laryngopharyngoesophagectomy combined with low AMT was performed under general anesthesia. The esophagus was mobilized under a thoracoscope, and the tubular stomach was created laparoscopically. After a Y-shaped incision was made in the cervical thorax, the anterior cervical muscles were dissected, and the thyroid gland was preserved. Afterward, the cervical systemic lymph node dissection was done. After total laryngectomy, the trachea was resected about $3 \mathrm{~cm}$ above the carina, and the trachea was intubated intraoperatively for ventilation. The sternoclavicular joint, the sternal stalk and the medial portions of rib cartilage were all removed, but the clavicle was preserved. Then, the tubular stomach was uplifted to the neck via the posterior mediastinal route for manual anastomosis. Double S-shaped pectoralis major musculocutaneous flap were harvested at the upper edge of the sternal stump for repairing the tracheal stump (Figure 4). A low AMT was performed on the chest. During this process, the trachea traveled inferior to the left innominate vein and right to the brachiocephalic trunk. The greater omentum was preserved for filling the anterior mediastinal area during the tubular stomach uplifting, while the bilateral sternocleidomastoid muscles were also used to fill the anterior mediastinal area. Thus, the tubular stomach 


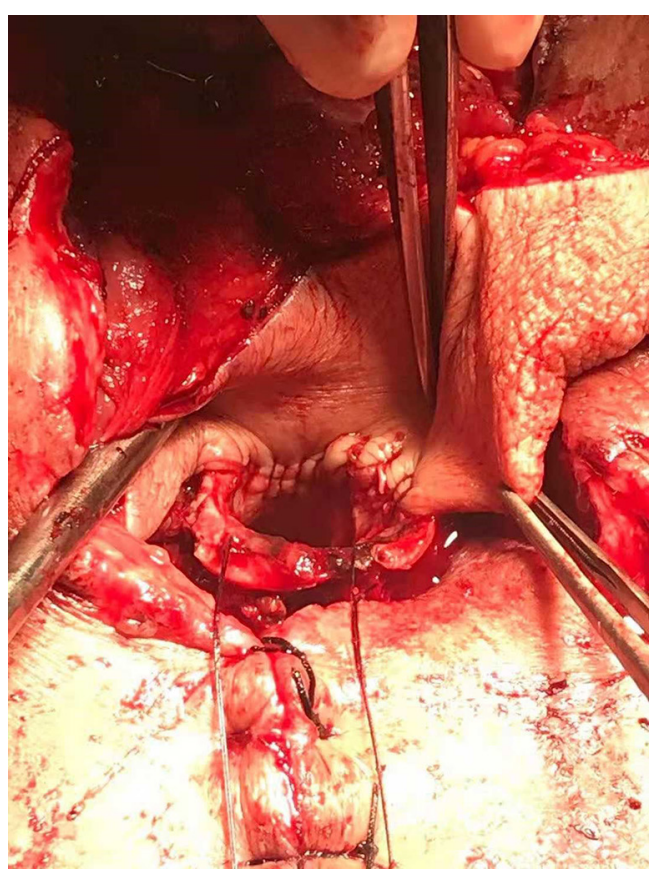

Figure 4 Double S-shaped myocutaneous flaps were harvested at the upper edge of the sternal stump for creating an anastomosis with the tracheal stump.

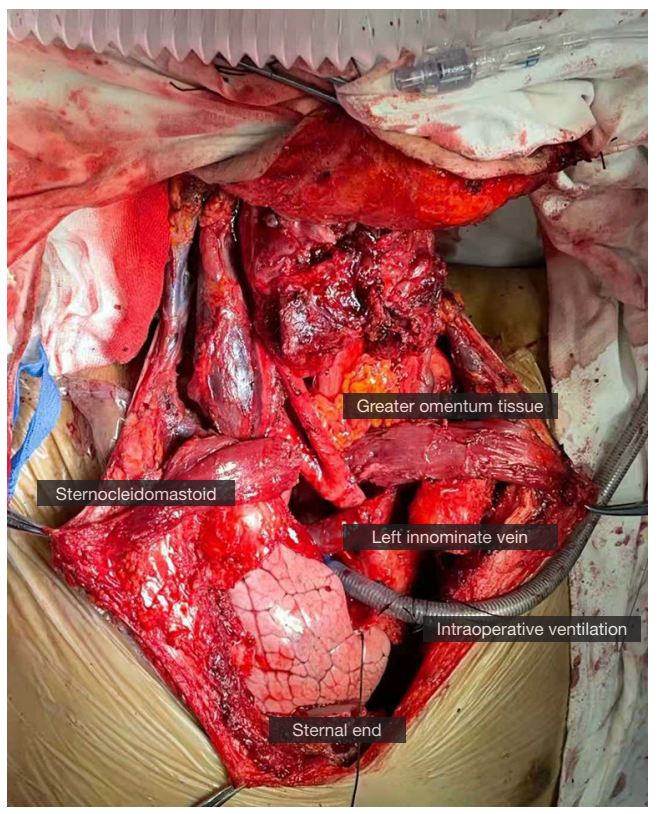

Figure 5 Intraoperative anatomy. The sternocleidomastoid and greater omentum were used for filling the anterior mediastinum. The trachea traveled inferior to the left innominate vein and right to the brachiocephalic trunk.

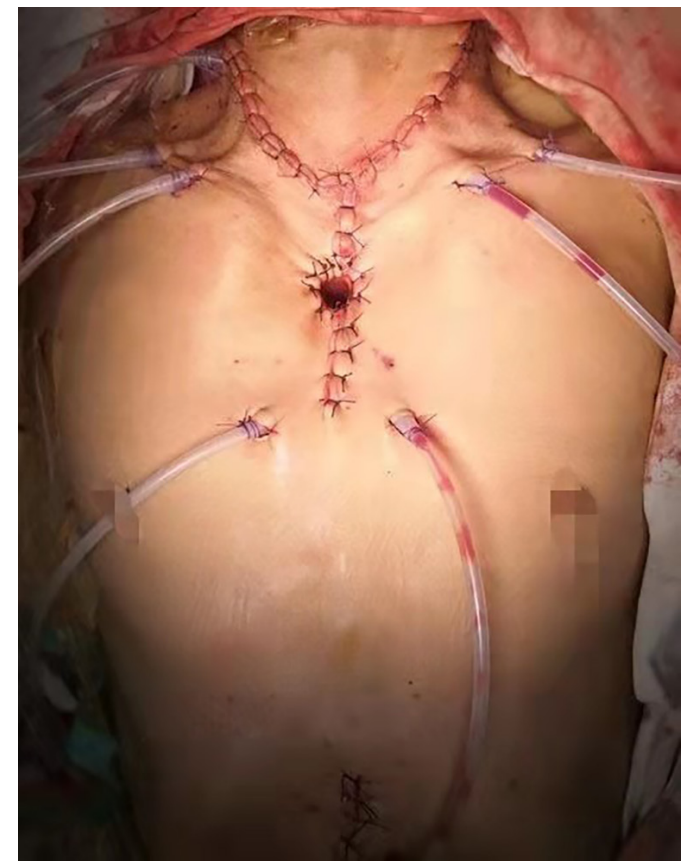

Figure 6 Negative-pressure drainage balls were placed on both sides of the anastomosis, above and below the tracheostomy and under the skin for adequate drainage.

was isolated from the trachea (Figure 5). Negative pressure drainage was placed on both sides of the anastomosis, above and below the tracheostomy and under the skin for good drainage (Figure 6). A second chest CT was performed on the tenth postoperative day, showing that the tracheostomy was well healed, and the mediastinal area was well filled with no cavity (Figure 7).

On postoperative day 7 , the patient was disconnected intermittently from the ventilator, and an artificial nose was used instead. Unfortunately, a minor anastomotic leakage appeared on postoperative day 8 . The cervical incision was opened, and wound dressings were changed twice a day. On day 10 , the patient was completely disconnected from the ventilator successfully, and a metal cannula was placed to prevent the tracheostomy closure. On day 27 , the patient was discharged smoothly.

The pathological result showed moderately/welldifferentiated squamous cell carcinoma of the esophagus, accompanied by necrosis, and sized about $5.5 \mathrm{~cm} \times 3.5 \mathrm{~cm}$. The tumor penetrated the esophageal wall and invaded the submucosa of the trachea. However, cancer tissue was negative in the upper-cut end of the larynx and esophagus, 

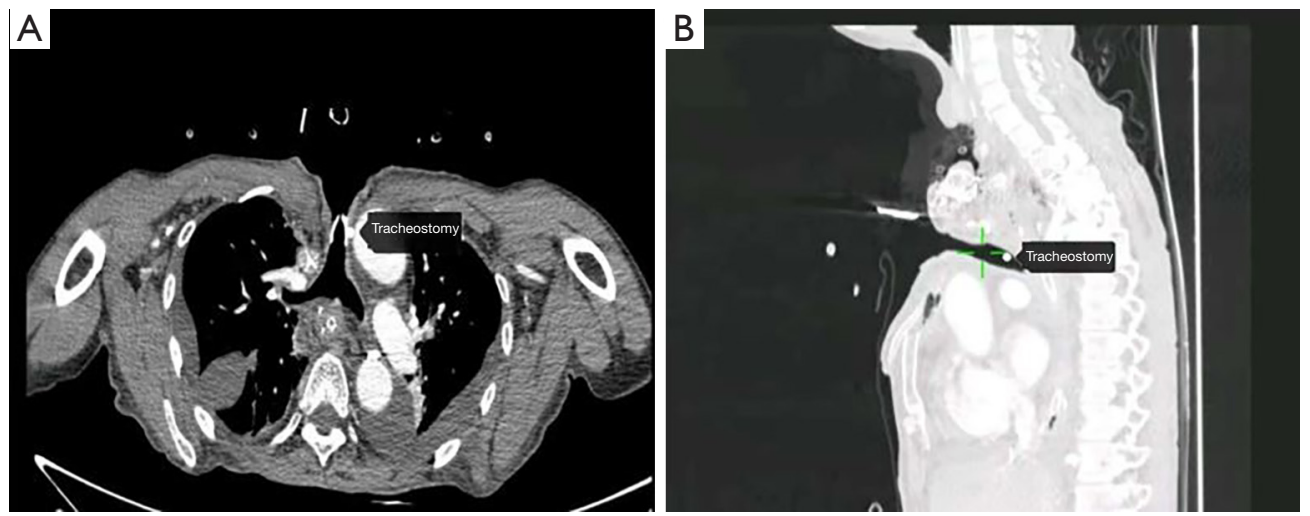

Figure 7 Postoperative CT images showed the trachestomy is good.

lower-cut end of the trachea, left/right/anterior resection margins of the larynx, and cut end of the cardia. Twenty lymph nodes were dissected totally, and no metastasis was observed. The pathological stage was $p T_{4 b} N_{0} M_{0}$. Up to now, the patient has survived 8 months after surgery without recurrence and metastasis.

\section{Discussion}

AMT was first reported in 1952 by Kleitsch (5) and Minor (6), respectively, for treating recurrent tracheal tumors. In 1959, Waddell and Cannon (7) described a technique for subtotal excision of the trachea and establishing a sternal tracheostomy in four patients. In the early years, the most serious postoperative complication resulting from this procedure was rupture and bleeding of the innominate artery. These complications were usually related to pressure necrosis of the innominate artery caused by the trachea, tracheostoma separation caused by tension suture leading to exposure of the great vessels particularly in patients with a prior history of radiation therapy, and persistence of dead space in the upper mediastinum because of mediastinitis or mediastinal abscess $(7,8)$. Waddell and Cannon (7) recommended moving the trachea below the innominate artery to avoid close contact of the tracheal stump with the innominate artery. To fill the dead space in the anterior mediastinum, Grillo (9) recommended sternotomy, including the resection of the manubrium sterni and the costal cartilages of the first and second ribs on either side. Full omentoplasty was performed for filling the anterior mediastinal cavity. To minimize the tension at the trachea-skin anastomosis, they used bilateral free pectoralis major flaps to construct the opening. These measures were effective in reducing the incidence of postoperative adverse events. Modifications continued in the coming decades. The technique proposed by Grillo has been widely adopted since the 1980s and became the golden standard for mediastinal tracheostomy. In honor of Grillo's contribution to this procedure, it was later named after him.

The indications of Grillo's technique include: (I) a subglottic tumor or the proximal tracheal cancer invading the subglottic larynx; (II) anastomotic recurrence after laryngectomy; (III) a highly differentiated thyroid cancer invading the trachea or recurring; and (IV) other benign tracheal lesions (10). Since Grillo's technique is a highly invasive procedure, the patients should have a good general condition, including satisfactory nutritional status. The distal trachea should not have cancerous tissue visible to the naked eye within at least $5 \mathrm{~cm}$ from the carina for cancer patients.

Few articles have described using low AMT to treat stage $\mathrm{T}_{4}$ esophageal cancer after neoadjuvant therapy, and the perioperative survival and long-term survival outcomes remain unknown. Although several studies in the 1990s evaluated the efficacy of AMT for esophageal cancer $(11,12)$, no literature has shown the value of AMT in the multidisciplinary treatment of $\mathrm{T}_{4}$ esophageal cancer. Yamasaki et al. (13) retrospectively analyzed the clinical and prognostic data of 27 consecutive patients with esophageal cancer with tracheal invasion who underwent AMT following neoadjuvant radiochemotherapy. Seventeen patients $(63 \%)$ had Clavien-Dindo grade I or higher complications and 12 patients (44\%) had grade III or higher complications. The in-hospital mortality rate was $4 \%$, and one patient died 48 days after surgery due to a ruptured brachiocephalic artery due to tracheal compression. The 30-day and 90-day mortality rates were 
$0 \%$ and $4 \%$, respectively. The 3 -year and 5 -year diseasefree survival rates and overall survival rate were $25.9 \%$, $18.5 \%$ and $38.6 \%, 25.7 \%$ respectively. Multivariate analysis demonstrated that response to prior treatment was an independent prognostic factor for these patients. Tracheal resection combined with AMT can be used as part of a multidisciplinary approach to the treatment of esophageal cancer involving the trachea.

In 2001, Kuwabara et al. (14) reported 7 cases of AMT, compared with his cases, the length of this patient's trachea invading is longer, thus the procedure is more technique demanding. In order to ensure that radical resection, we innovatively adopted the application of Double S-shaped myocutaneous flaps, which not only helps to extend the trachea fixed to the chest wall, but also helps to reduce the possibility of the necrosis of trachea membrane.

The biggest postoperative risk of AMT is unmanageable hemorrhage caused by large blood vessels due to the infection behind of the trachea. At present, the commonly used solutions are the local space packing of the omentum or thoracic myocutaneous flap to reduce local space and effusion. In terms of the lower AMT for this patient, we have to removed part of clavicle, upper sternum combined with the first and second costal cartilage at the same time to avoid fluid accumulation, which is conducive to local infection. The new method used in this patient is satisfactory and no infection of the anterior mediastinum occurred. Fortunately, this patient discharged smoothly.

In conclusion, our current case also showed that low AMT is associated with tolerable perioperative outcomes as part of the multidisciplinary treatment of locally advanced esophageal cancer at the cervicothoracic junction with tracheal invasion. Improving AMT safety by important surgical tips as mentioned above is very useful to reduce postoperative fatal complications, and optimizing patient selection may improve the prognosis among these patients.

\section{IMDT discussion}

\section{Department of Oncology}

The patient was clinically diagnosed with esophageal cancer at the cervicothoracic junction, with long-segmental tracheal membrane involvement possible. The biopsy showed esophageal squamous cell carcinoma. PETCT and chest CT showed no mediastinal lymph node metastasis or distant metastasis, thus the clinical stage was $c \mathrm{~T}_{4} \mathrm{~N}_{0} \mathrm{M}_{0}$. The tumor was borderline resectable due to its invasive relationship to the trachea. The initial treatment should be neoadjuvant chemotherapy or neoadjuvant concurrent chemoradiotherapy. The tissue fibrosis caused by radiotherapy might have a disadvantageous impact on surgery, especially on laryngeal preservation, therefore docetaxel plus 5-fluorouracil and cisplatin (DCF) neoadjuvant chemotherapy may be preferable. The subsequent therapeutic strategy could be made according to the treatment response.

\section{Department of Esophageal Surgery}

The patient received neoadjuvant chemotherapy and the efficacy was SD. Lymph nodes are in good condition with indications for surgery. We believe that resection of R0 tumor can bring benefits to the survival of patients, so combined organ resection is involved in the operation, and we consider a low-AMT operation to remove the involved trachea. In addition, the trachea is moved down to the left innominate vein and to the right to the cephalic and brachial trunk during the operation to prevent postoperative innominate artery bleeding. The anterior mediastinal area with omentum filling and bilateral sternocleidomastoid filling were retained during tubular gastric eminences. At the same time, attention should be paid to the blood supply of the flap at the stoma.

\section{Department of Anesthesiology}

Currently, her cardiopulmonary function was good, with a PS score of 1 . The patient had a previous history of tongue cancer resection. An adequate preoperative airway assessment should be performed to evaluate whether there was difficult tracheal intubation or not during the operation. Tracheal intubation should be performed on the operating table for ventilation if the patient received AMT. Meanwhile, blood gases should be carefully monitored during the surgery.

\section{The following questions are for international experts}

Question 1: How to get an accurate clinical stage before treatment for cervicothoracic esophageal cancer? Regarding the borderline resectable lesion, how can we evaluate whether the tumor invades the trachea?

Raja Flores: A combination of PET scan, CT scan with contrast and bronchoscopy/esophagoscopy/endoscopic ultrasound. 
Biniam Kidane: Accurate clinical staging can be achieved with PET-CT, Diagnostic neck/chest CT, EGD, EUS and flexible bronchoscopy.

Masatsugu Hamaji: This is a challenging question. I am uncertain if we have better modalities than CT, PET, esophagogastroduodenoscopy, and bronchoscopy.

Regarding evaluation of whether the tumor invades the trachea, intraoperative macroscopic and microscopic evaluation would be a gold standard.

Question 2: dCRT is the current standard of care for cervical esophageal cancer, as listed in the NCCN guideline. However, salvage surgery is useful for some residual or recurrent lesions even if it has a high risk. So, could we choose DCF neoadjuvant chemotherapy for cervical esophageal cancer? And what are the advantages and disadvantages compared to dCRT? Raja Flores: Either one is ok. DCF or Bdcf.

Biniam Kidane: Compared to dCRT, the major advantage of neoadjuvant chemotherapy is that it facilitates less complicated surgical resection and also preserves radiotherapy for use in the future for recurrent disease. The major disadvantage in use of neoadjuvant chemotherapy is the reduced probability of inducing complete pathologic response as well as the increased intensity of the cytotoxic chemotherapeutic regimen.

Masatsugu Hamaji: The authors should first state the guidelines and indications on neoadjuvant chemoradiotherapy. Salvage surgery should be differentiated from resection after neoadjuvant. The advantages and disadvantages are currently unknown and should be analyzed on the basis of retrospective clinical studies.

Question 3: As we know, immunotherapy has been shown to bring good treatment results for locally advanced esophageal cancer and in neoadjuvant treatment combined with chemotherapy. Then, can we try to use a stronger systemic treatment, such as immune checkpoint inhibitor plus with chemo, to replace chemoradiotherapy as neoadjuvant therapy for clinical borderline resectable cervical esophageal cancer?

Raja Flores: Yes, and repeat the scans to determine response pre surgery.

Biniam Kidane: Although there have been good results with treatment of squamous cell cancers in the metastatic setting and although there is good preclinical evidence and plausible biological rationale for use of combination cytotoxic chemotherapy and immune checkpoint inhibitor therapy as a neo-adjuvant therapy, there is no reliable evidence to guide support it currently. Thus, this would be something to address in the context of a clinical trial. However, if data in related cancers is any indication, there is great promise in this space.

Masatsugu Hamaji: This is also a challenging question. The authors are strongly encouraged to refer to clinical trials on "definitive chemoradiotherapy and immunotherapy" or "induction chemoradiotherapy and immunotherapy".

\section{Acknowledgments}

Funding: None.

\section{Footnote}

Reporting Checklist: The authors have completed the CARE reporting checklist. Available at https://dx.doi. org/10.21037/jgo-21-685

Conflicts of Interest: All authors have completed the ICMJE uniform disclosure form (available at http://dx.doi. org/10.21037/jgo-21-685). The authors have no conflicts of interest to declare.

Ethical Statement: The authors are accountable for all aspects of the work in ensuring that questions related to the accuracy or integrity of any part of the work are appropriately investigated and resolved. All procedures performed in this study involving human participants were in accordance with the ethical standards of the institutional and/or national research committee(s) and with the Helsinki Declaration (as revised in 2013). Written informed consent was obtained from the patient for publication of this case report and accompanying images. A copy of the written consent is available for review by the editorial office of this journal.

Open Access Statement: This is an Open Access article distributed in accordance with the Creative Commons Attribution-NonCommercial-NoDerivs 4.0 International License (CC BY-NC-ND 4.0), which permits the noncommercial replication and distribution of the article with the strict proviso that no changes or edits are made and the 
original work is properly cited (including links to both the formal publication through the relevant DOI and the license). See: https://creativecommons.org/licenses/by-nc-nd/4.0/.

\section{References}

1. Ikeda K, Ishida K, Sato N, et al. Chemoradiotherapy followed by surgery for thoracic esophageal cancer potentially or actually involving adjacent organs. Dis Esophagus 2001;14:197-201.

2. Noguchi T, Moriyama H, Wada S, et al. Resection surgery with neoadjuvant chemoradiotherapy improves outcomes of patients with T4 esophageal carcinoma. Dis Esophagus 2003;16:94-8.

3. Malaisrie SC, Untch B, Aranha GV, et al. Neoadjuvant chemoradiotherapy for locally advanced esophageal cancer: experience at a single institution. Arch Surg 2004;139:5328; discussion 538-9.

4. de Manzoni G, Pedrazzani C, Pasini F, et al. Chemoradiotherapy followed by surgery for squamous cell carcinoma of the thoracic esophagus with clinical evidence of adjacent organ invasion. J Surg Oncol 2007;95:261-6.

5. Kleitsch WP. Anterior mediastinal tracheotomy. J Thorac Surg 1952;24:38-42.

6. Minor GR. Trans-sternal tracheal excision for carcinoma; report of a case. J Thorac Surg 1952;24:88-92.

7. Waddell WR, Cannon B. A technic for subtotal excision

Cite this article as: Chen C, Flores R, Kidane B, Hamaji M, Guo X. Application of low anterior mediastinal tracheostomy for locally advanced cervicothoracic esophageal cancer undergoing total laryngopharyngoesophagectomy: a case report. J Gastrointest Oncol 2021;12(6):3107-3114. doi: 10.21037/ jgo-21-685 of the trachea and establishment of a sternal tracheostomy. Ann Surg 1959;149:1-8.

8. Sisson GA, Straehley CJ Jr, Johnson NE. Mediastinal dissection for recurrent cancer after laryngectomy. Laryngoscope 1962;72:1064-77.

9. Grillo HC. Terminal or mural tracheostomy in the anterior mediastinum. J Thorac Cardiovasc Surg 1966;51:422-7.

10. Conti M, Benhamed L, Mortuaire G, et al. Indications and results of anterior mediastinal tracheostomy for malignancies. Ann Thorac Surg 2010;89:1588-95.

11. Orringer MB. Anterior mediastinal tracheostomy with and without cervical exenteration. Ann Thorac Surg 1992;54:628-36; discussion 636-7.

12. Kato H, Tachimori $Y$, Watanabe $H$, et al. Mediastinal tracheostomy during esophagectomy for cervicothoracic esophageal carcinoma invading the proximal trachea. J Surg Oncol 1994;55:78-83.

13. Yamasaki M, Yamashita K, Saito T, et al. Tracheal resection and anterior mediastinal tracheostomy in the multidisciplinary treatment of esophageal cancer with tracheal invasion. Dis Esophagus 2020;33:doz101.

14. Kuwabara Y, Sato A, Mitani M, et al. Use of omentum for mediastinal tracheostomy after total laryngoesophagectomy. Ann Thorac Surg 2001;71:409-13.

(English Language Editor: J. Chapnick) 